\title{
The Impact of Body Mass Index on Functional Rehabilitation Outcomes of Working-age Inpatients with Stroke
}

\author{
Alejandro Garcia-Rudolph \\ Universitat Autonoma de Barcelona \\ John D. Kelleher \\ Technological University Dublin, john.d.kelleher@tudublin.ie \\ Blanca Cegarra \\ Universitat Autonoma de Barcelona
}

See next page for additional authors

Follow this and additional works at: https://arrow.tudublin.ie/q4articles

Part of the Medicine and Health Sciences Commons

\section{Recommended Citation}

Garcia-Rudolph, A. et al. (2020). The impact of body mass index on functional rehabilitation outcomes of working-age inpatients with stroke. European Journal of Physical and Rehabilitation Medicine, December. doi:0.23736/S1973-9087.20.06411-4

This Article is brought to you for free and open access by the Precise4Q at ARROW@TU Dublin. It has been accepted for inclusion in Articles by an authorized administrator of ARROW@TU Dublin. For more information, please contact arrow.admin@tudublin.ie, aisling.coyne@tudublin.ie,gerard.connolly@tudublin.ie.

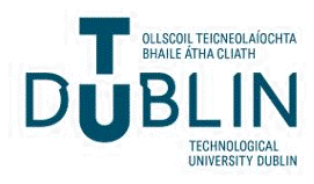




\section{Authors}

Alejandro Garcia-Rudolph, John D. Kelleher, Blanca Cegarra, Joan Sauri Ruiz, Vasudevan Nedumpozhimana, Eloy Opissso, Jose M. Tormos, and Montserrat Bernabeu 
Title: The Impact of Body Mass Index on Functional Rehabilitation Outcomes of Working-age Inpatients with Stroke.

Alejandro García-Rudolph ${ }^{1,2,3^{*}}$, John D. Kelleher ${ }^{5}$, Blanca Cegarra ${ }^{1,2,3,4}$, Joan Saurí Ruiz ${ }^{1,2,3}$, Vasudevan Nedumpozhimana ${ }^{5}$, Eloy Opissso ${ }^{1,2,3}$, Josep Maria Tormos ${ }^{1,2,3}$, Montserrat Bernabeu ${ }^{1,2,3}$

1-Department of Research and Innovation, Institut Guttmann, Institut Universitari de Neurorehabilitació adscrit a la UAB, Badalona, Barcelona, Spain;

2-Universitat Autònoma de Barcelona, Bellaterra (Cerdanyola del Vallès), Spain;

3-Fundació Institut d'Investigació en Ciències de la Salut Germans Trias i Pujol, Badalona, Barcelona, Spain

4- Universitat de Barcelona, Barcelona, Spain

5- Information, Communication and Entertainment Research Institute, Technological University Dublin (TU)

\section{Corresponding author}

Alejandro García-Rudolph

Department of Research and Innovation

Institut Guttmann - Hospital de Neurorehabilitació

Cami Can Ruti s/n 08916 - Badalona- Barcelona - Spain

Tel: +3493497 $7700-2265$

Fax: +34 934977707

Email: alejandropablogarcia@gmail.com 


\section{Running head: body mass index on stroke recovery}

Words count: 3695

\section{Acknowledgements}

Special thanks to Jaume Lopez Carballo from the Research Department of Institut Guttmann.

\section{Financial Support}

This research was partially funded by H2020 Personalised Medicine by Predictive Modeling in Stroke for better Quality of Life (PRECISE4Q) Grant Nr 777107

\section{Disclosure of interest}

The authors report no conflict of interest

\section{Ethical Standards}

The authors assert that all procedures contributing to this work comply with the ethical standards of the relevant national and institutional committees on human experimentation and with the Helsinki Declaration of 1975, as revised in 2008 
Title: The Impact of Body Mass Index on Functional Rehabilitation Outcomes of Working-age Inpatients with Stroke.

\begin{abstract}
Background: Stroke is the most relevant cause of acquired persistent disability in adulthood.

The relationship between patient's weight during rehabilitation and stroke functional outcome is controversial, previous research reported positive, negative and no effects, with scarce studies specifically addressing working-age patients.
\end{abstract}

Aim: To evaluate the association between Body Mass Index (BMI) and the functional progress of adult ( $<65$ years) patients with stroke admitted to a rehabilitation hospital.

Design: Retrospective observational cohort study.

Setting: Inpatient rehabilitation center.

Population: 178 stroke patients (ischemic or hemorrhagic).

Methods: Point-biserial and Spearman's correlations, multivariate linear regressions and analysis of covariance were used to describe differences in functional outcomes after adjusting for age, sex, severity, dysphagia, depression and BMI category. Functional Independence Measure (FIM), FIM gain, efficiency and effectiveness were assessed.

Results: Participants were separated in 3 BMI categories: normal weight (47\%), overweight (33\%) and obese (20\%). There were no significant differences between BMI categories in any functional outcome (total FIM (TFIM), cognitive (CFIM), motor (MFIM)) at discharge, admission, gain, efficiency or effectiveness.

In regression models BMI (as continuous variable) was not significant predictor of TFIM at discharge after adjusting for age, sex, severity, dysphagia, depression and ataxia $\left(\mathrm{R}^{2}=0.4813\right)$, significant predictors were TFIM at admission $(\beta=0.528)$ and NIHSS $(\beta=-0.208)$. 
MFIM efficiency did not significantly differ by BMI subgroups, neither did CFIM efficiency. Length of stay (LOS) and TFIM effectiveness were associated for normal ( $\mathrm{r}=0.33)$ and overweight $(\mathrm{r}=0.43)$, but not for obese. LOS and TFIM efficiency were strongly negatively associated only for obese ( $\mathrm{r}=-0.50)$.

Conclusions: FIM outcomes were not associated to BMI, nevertheless each BMI category when individually considered (normal weight, overweight or obese) was characterized by different associations involving FIM outcomes and clinical factors.

Clinical Rehabilitation Impact: In sub-acute post-stroke working-age patients undergoing rehabilitation, BMI was not associated to FIM outcomes (no obesity paradox was reported in this sample).

Distinctive significant associations emerged within each BMI category, (supporting their characterization) such as length of stay and TFIM effectiveness were associated for normal weight and overweight, but not for obese. Length of stay and TFIM efficiency were strongly negatively associated only for obese.

Keywords: stroke, body mass index; independent living; rehabilitation 


\section{Introduction.}

Stroke is a leading cause of acquired disability in adults worldwide ${ }^{1}$ with excess body weight being an acknowledged risk factor for stroke ${ }^{2}$. Nevertheless, the effect of obesity on stroke clinical outcomes is unclear, with previous studies reporting lower levels of impairment and mortality in obese patients compared with normal weight patients ${ }^{1}$. In 2002 , Gruberg and colleagues introduced the obesity paradox concept, in the context of coronary artery disease, for describing that overweight and obese patients had (paradoxically) better outcomes than normal weight patients ${ }^{3}$. Similar findings were reported in the context of other chronic diseases and therefore the use of the term has become widespread ${ }^{1}$. Nevertheless, stroke outcomes have been widely conflicting in that regard and explanations currently remain ambiguous $^{4}$. A recent narrative review ${ }^{1}$ supports the existence of the obesity paradox in stroke, concluding that most studies reported lower mortality levels and better functional outcomes in obese and overweight patients than in normal weight and underweight patients. However, authors remarked that this is still controversial and further higher quality evidence is needed ${ }^{1}$.

Specifically regarding functional recovery, in a recent systematic review MacDonald and coallegues $^{5}$ concluded that based on the current evidence it is unclear whether functional outcomes of adults undergoing inpatient stroke rehabilitation, are affected by obesity. All studies classified obesity using Body Mass Index (BMI) and most of them used the Functional Independence Measure (FIM) as outcome measure. 
Furthermore, to our best knowledge, existing studies have scarcely considered potential confounders previously related to functional outcomes, such as diabetes ${ }^{6}$, depression ${ }^{7}$, atrial fibrillation $^{8}$, ataxia ${ }^{9}$, dysphagia $^{10}$, hypertension ${ }^{11}$ or recurrent stroke $e^{12}$.

Besides, associations between factors for ischemic or hemorrhagic stroke and clinical outcomes have been analyzed predominantly in older rather than younger patients, e.g. the mean age across studies included in McDonalds ${ }^{5}$ review ranged from 63 to 82 . Nevertheless, the incidence of any stroke in the young (18-44 years) has increased by $23 \%$ during the past ten years ${ }^{13}$. Ischemic stroke is no longer a disease affecting just elderly people with an estimated 3.6 million young people (age<55 years) affected each year ${ }^{14}$.

As reported in previous research, in elderly patients (age $>70 \mathrm{yrs}$ ), excess body weight might have a protective effect ${ }^{15}$. Furthermore, age and stroke severity are the most powerful predictors of stroke outcome ${ }^{1}$.

In this study we propose to evaluate the influence of the patient's weight, measured as BMI, on rehabilitation functional outcomes, measured using the FIM, in first event or recurrent stroke, working-age (mostly severe) patients in sub-acute rehabilitation. To that aim we are using variables identified in previous research such as stroke severity, measured using the National institute of Health Stroke Scale (NIHSS), gender, age, total FIM (T-FIM), motor FIM (M-FIM) and cognitive FIM (C-FIM), further extending them with specific clinical factors (diabetes, atrial fibrillation, dysphagia, ataxia and depression) scarcely addressed in previous research 


\section{Methods.}

\subsection{Study Design.}

The data that support the findings of this study are available from the corresponding author upon reasonable request. We conducted a retrospective observational cohort study enrolling subacute ischemic or hemorrhagic patients with stroke admitted to the Rehabilitation Unit of the Acquired Brain Injury Department of XX (anonymized) hospital. Recruitment period was from March 2012 to October 2019.

This study conforms to the STROBE Guidelines ("Strengthening the Reporting of Observational Studies in Epidemiology") ${ }^{16}$.

\subsection{Participants.}

Eligible participants were adult patients $(\geq 18$ and $\leq 64$ y.o.) with the diagnosis of first-time or recurrent stroke, receiving inpatient subacute rehabilitation and with electronical health records including complete data within 10 days of admission.

Patients were excluded for the following reasons: major musculoskeletal problems, more than 3 weeks of the onset of symptoms since admission to inpatient subacute rehabilitation, cases of transient ischemic attack or subarachnoid hemorrhage, diagnosis of stroke in the context of another concomitant comorbidity (e.g. traumatic brain injury) and a previous history of another disabling condition.

\subsection{Functional Assessments.}

A physician assessed functional status using the FIM. The FIM scale includes 18 items structured in 2 domains: the motor domain, including 13 items, and the cognitive domain, including 5 items. The total score is obtained by adding the motor score (range 3-91) to the 
cognitive score (range 5-35) and ranges from 18 to 126 with a higher score indicating a higher degree of independence.

FIM gain is defined as the difference between FIM at admission and FIM at discharge. FIM efficiency is defined as FIM gain divided by LOS.

Effectiveness is defined as: (final score-initial score)/(maximum score-initial score) $\times 100^{17}$.

The RPG (Rehabilitation Patient Groups) benchmark ${ }^{18}$ is used in this work to stratify patients based on age and functional ability measured using the MFIM at admission and CFIM at admission. The RPG classifies stroke severity as mild-RPG, moderate-RPG or severe-RPG (details of the RPG algorithm are presented in Supplementary Material, Figure SM1).

\subsection{Clinical variables.}

At hospital admission, stroke severity was assessed using the NIHSS. Medical complications and comorbidities (reported using ICD9 codes) were collected from the participants' electronical health records (EHRs). The following were included as predictor variables: diabetes, dysphagia, depression, hypertension, smoking habits and atrial fibrillation (all of them recorded as yes/no). Missing values were completed by means of the specific internal or external reports.

Demographics (age, sex, education), stroke characteristics (type, and location), time since stroke onset to rehabilitation admission after discharge from an acute stroke care facility (in days), were also obtained from EHRs, as well as BMI at admission.

Patients were separated into 4 groups according to their BMI at admission using the World Health Organization (WHO) classification system ${ }^{5}$ : underweight: $<18.5 \mathrm{~kg} / \mathrm{m}^{2}$; normal weight: 18.51 to $25 \mathrm{~kg} / \mathrm{m}^{2}$; overweight: 25.1 to $30 \mathrm{~kg} / \mathrm{m}^{2}$ and obese $>30 \mathrm{~kg} / \mathrm{m}^{2}$. 


\subsection{Rehabilitation program}

All patients were admitted to the Rehabilitation Unit of our Acquired Brain Injury Department and underwent motor and cognitive rehabilitation - starting usually the day after admission and lasting until discharge. The rationale for motor and cognitive rehabilitation was based on currently available knowledge ${ }^{19}$ and hospital protocols.

\subsection{Statistical Analyses.}

All statistical analyses were performed in R-v3.5.1 (64 bits), level of significance was set at $\mathrm{p}=0.05$. Patients were stratified into four groups according to their BMI as described in section 2.4. Descriptive statistics were used for demographic and clinical characteristics of participants as well as functional assessments. The four groups were compared using the $\chi^{2}$ test for categorical variables and the Kruskal-Wallis test for continuous and ordered variables.

FIM at discharge and FIM efficiency were analyzed using two different approaches (for comparison with previous research).

The bivariate (Spearman's) correlation was used to evaluate the association between FIM outcomes (efficiency and FIM at discharge) and BMI (as continuous variable). Correlated variables $(\mathrm{p}<0.05)$ were submitted as independent variables to the multivariate analysis (TFIM at discharge as dependent variable). Categorical variables were dichotomized (yes =1, no=0; woman $=0, \operatorname{man}=1$; current smoker $=1$, former smoker and nonsmoker $=0$; less than 12 years of education $=0$, more than 12 years of education $=1$ ).

Multicollinearity of independent variables is tested by the variance inflation factor (VIF) and the tolerance (1/VIF). Tolerance is associated with each independent variable and ranges from 0 to 1 . A tolerance below 0.40 and/or a VIF of 5 and above indicates a multicollinearity 
problem $^{20}$. The assumption of independent errors is evaluated using the Durbin-Watson. The closer to 2 that the value is, the better. As a conservative rule it is suggested that for values less than 1 or greater than 3 the assumption of independence is not met $^{20}$. Analysis of covariance was used to describe differences in FIM scores after adjusting for age, sex, and weight group. A FIM efficiency adjusted mean was estimated for each of the 4 weight categories. The pairwise comparisons between the 4 weight groups were completed using the Tukey honestly significant difference test. An adjusted FIM efficiency mean for the 4 weight categories was defined as the predicted response value obtained by evaluating the regression equation for each weight category at the mean for the other covariates included in the regression model. Multivariable regression analysis of FIM efficiency scores was performed by regressing the FIM efficiency discharge score on the FIM admission score adjusted for BMI as a continuous covariate and adjusted for sex, age, and length of hospital stay.

\subsection{Ethical considerations.}

The study follows the Declaration of Helsinki and this study was approved by the Ethics Committee of Clinical Research of XX (anonymized). The participants are anonymized and non-identifiable. 


\section{Results}

The source population was the total number of stroke patients admitted to the rehabilitation unit of the XXX (omitted) hospital during the whole period under study (March 2012 to October 2019).

A total of 1217 stroke patients composed such population. After excluding 158 patients with more than 3 weeks since stroke onset to admission and 137 patients with more than one week since admission to FIM assessment, 922 were analyzed for FIM at discharge. After excluding 198 patients with more than one week since discharge to FIM assessment 724 were included for FIM gain calculation.

After excluding 97 with missing T-FIM, 193 with missing C-FIM, 189 with missing M-FIM, 34 were removed in relation to demographics or clinical data (e.g the total number of underweight participants was 3 therefore they were removed and this BMI category was excluded from the analysis) 251 patients were analyzed in relation to acute NIHSS, 73 of them were not available, leaving 178 patients (The patient selection flowchart is presented in Supplementary material Figure SM2).

All participants included in our study have been admitted in a stroke unit receiving appropriate acute treatment, most of them supported by the Stroke Code System in Catalonia. As shown in Table SM1, 96\% of participants in this study come from Catalonia. The Stroke Code System (SCI-Cat) implemented since 2006, is set in motion when a person suffers a stroke and the same patient or someone in their environment alerts the healthcare system. The SCI-Cat guarantees the urgent and priority transfer of the patient to the nearest hospital with the appropriate diagnostic and therapeutic capacity. 
Table 1 summarizes the demographic and clinical characteristics at admission by BMI categories, these data demonstrate that $47 \%$ were normal weight, $33 \%$ were overweight, and $20 \%$ were obese. The mean BMIs for the same categories were $22.3 \mathrm{~kg} / \mathrm{m}^{2}, 27.0 \mathrm{~kg} / \mathrm{m}^{2}$, and $32.1 \mathrm{~kg} / \mathrm{m}^{2}$, respectively.

The participant's mean age was 49.6 with no significant differences between them (mean age was $48.5,50.7$ and 50.6 respectively), $31.5 \%$ were women $(34.5 \%, 16.9 \%$ and $48.66 \%)$ $(\mathrm{p}=0.004)$.

The average number of days since stroke onset to rehabilitation admission was $17.76 \pm 4.59$. As shown in Table 1, there is a majority of ischemic stroke participants $(71.3 \%)$ and according to Oxfordshire Community Stroke Project (OCSP) classification, more than half of them were total anterior circulation infarcts (TACI) in all three BMI categories.

There were no statistically significant differences between groups in the admission NIHSS total score. (mean values were $12.96 \pm 5.49,12.40 \pm 5.44$ and $13.08 \pm 5.05$ respectively).

There were no statistically significant differences between groups either when considering RPG severity, nevertheless as shown in Table 1, in all three BMI categories half of the participants are classified as RPG-severe. In particular in the obese BMI category $57.1 \%$ of participants are RPG-severe. Only $14.6 \%$ of all participants are classified as RPG-mild.

The percentage of RPG-severe patients across BMI categories was $51.7 \%$.

[Table 1]

\subsection{Functional assessments}

There were no statistically significant differences between groups at admission in the TFIM $(p=0.592)$, CFIM $(p=0.105)$, MFIM $(p=0.557)$; either at discharge TFIM $(p=0.857)$, CFIM 
$(p=0.229)$, MFIM $(p=0.436)$. Neither regarding FIM gain, efficiency, effectiveness for TFIM, CFIM, MFIM, as shown in Table 2.

[Table 2]

However some tendencies can be seen, as illustrated in Figure SM3 in Supplementary Material, the highest CFIM at admission and discharge can be seen in the obese group, intermmediate values correspond to the overweight group and lowest to the normal group. Similar behaviour can be seen in the CFIM effectiveness, though any of them is statistically signifficant (noted using "NS") .

\subsection{Correlations analyses}

We performed the bivariate Spearman's correlation analysis to evaluate the association between BMI (as a continuous variable) and TFIM at discharge, CFIM efficiency and MFIM efficiency. As shown in Table 3, BMI was not significantly associated to any of them.

We further explored Spearman's correlations between BMI and the following FIM assessments: TFIM at admission, TFIM at discharge, TGIM gain, TFIM efficiency, TFIM effectiveness, MFIM at admission, MFIM at discharge, MGIM gain, MFIM efficiency, MFIM effectiveness, CFIM at admission, CFIM at discharge, CGIM gain, CFIM efficiency, CFIM effectiveness.

None of them was significant, details are presented in Appendix.

[Table 3] 
Table 3 also shows the associations between TFIMDis, MFIM efficienciy and CFIM efficiency with other potential predictors, for dichotomous variables (e.g. Diabetes) we used point-biserial correlation. Such significant associations were entered as independent variables into the multiple regression model (TFIM at discharge as dependent variable) presented in Section 3.3.

\subsection{Multivariant analysis}

Results of regression analyses with TFIM at discharge as dependent variable is presented in Table 4 model 2 (after adjustment for age, sex and TFIM-admission) and BMI was not significantly associated with TFIM at discharge $(\mathrm{p}=0.264)\left(\mathrm{R}^{2}=0.4427\right)$

Table 4 model 1 uses potential predictors of TFIM at discharge obtained from Table 3, outperforming model $2\left(\mathrm{R}^{2}=0.4813\right)$ and identifying the following significant predictors: TFIM at admission $(\beta=0.528, \mathrm{p}<0.0001)$ and NIHSS $(\beta=-0.208, \mathrm{p}=0.003)$. When including BMI as independent variable in model 1 it was not found significant either.

Table 4 shows VIF for all predictors (are all well below 5), none of them close to 3 . Based on these measures we can safely conclude that there is no collinearity within our data.

[Table 4]

\subsection{ANCOVA analyses}

Tables 5 provides adjusted means for the MFIM efficiency and CFIM efficiency cognitive by weight category. After adjusting for age and sex, the MFIM efficiency did not significantly differ by BMI subgroups ( $\mathrm{p}=0.949)$ (Table 5 model 1$)$. 
After adjusting for age and LOS, the MFIM efficiency did not significantly differ by BMI subgroups $(\mathrm{p}=0.890)$ (Table 5 model 2).

After adjusting for age and sex, the CFIM efficiency did not significantly differ by BMI subgroups ( $\mathrm{p}=0.771)$ (Table 5 model 3).

After adjusting for NIHSS and dysphagia, the MFIM efficiency did not significantly differ by BMI subgroups ( $\mathrm{p}=0.902)$ (Table 5 model 4).

[Table 5]

\subsection{Correlation analysis within each BMI category}

We performed Spearman's correlation analyses separately for normal weight (presented in Table 6 top), overweight (Table 6 middle) and obese (Table 6 bottom) patients, to identify associations existing in one BMI category that do not exist in the others, for TFIM.

Significant correlations involving our main continuous variables (BMI, NIHSS, Age, LOS) and TFIM outcomes (TFIM gain, TFIM efficiency and TFIM effectiveness) are highligted in Table 6, showing different associations between them for the different BMI categories.

For example, LOS is significantly correlated to TFIM gain for the normal $(r=0.33, p=0.01)$ and overweight $(\mathrm{r}=0.43, \mathrm{p}<0.001)$ BMI categories, but it is non-significant for the obese category. LOS is also significantly correlated to TFIM effectiveness for the normal $(r=0.33$, $\mathrm{p}=0.01)$ and overweight $(\mathrm{r}=0.43, \mathrm{p}<0.001) \mathrm{BMI}$ categories, but it is non-significant for the obese category.

As shown in Table 6, TFIM at admission is the only variable correlated to TFIM gain for obese patients. This correlation between TFIM at admission and TFIM gain is stronger in 
normal $(r=-0.63, p<0.001)$ and in overweight patients $(r=-0.62, p<0.001)$ than in obese $(r=-$ $0.43, \mathrm{p}=0.01$ ) as shown in Table 6 .

In the normal weight patients, NIHSS is correlated to TFIMgain $(r=0.26, p<0.05)$ and TFIM effectiveness $(\mathrm{r}=0.26, \mathrm{p}<0.05)$ meanwhile for overweight and obese patients none of them are-significant.

In Supplementary Material Figure SM5 we included the analysis for CFIM showing similar results.

[Table 6] 


\section{Discussion.}

The relationship between patient's weight during rehabilitation and stroke functional outcome is controversial. MacDonald et $\mathrm{al}^{5}$ recently published a systematic review of the impact of obesity on stroke inpatient rehabilitation functional outcomes $(2,765$ titles and abstracts were screened and 64 articles were reviewed in full text). A total of seven studies (involving 3070 participants) met the inclusion criteria. Of the seven studies, two reported a positive

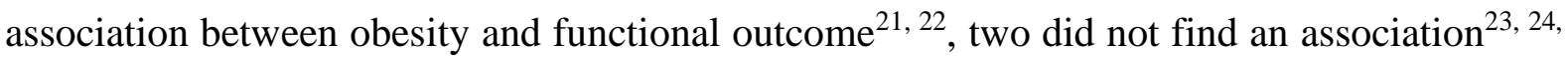
and three reported a negative association ${ }^{25-27}$. Five of the seven studies used FIM as their outcome measure.

Therefore our results are in keeping with those of Hagii et $\mathrm{al}^{23}$. and Karaahmet et $\mathrm{al}^{24}$. However, they did not use a BMI categorization and instead examined all individuals who were classified as overweight $(\mathrm{BMI} \geq 25 \mathrm{~kg} / \mathrm{m} 2)$. Hagii et $\mathrm{al}^{23}$ reported results using the Modified Rankin Scale (mRS) ${ }^{28}$. Nevertheless, mRS and FIM are highly correlated as reported in previous research ${ }^{29}$.

Karaahmet et $\mathrm{al}^{24}$ was one of the smallest studies included in MacDonald's review ${ }^{5}$, with 85 participants.

In this study we followed the WHO categorization, which has been also used in studies such as Burke et $\mathrm{al}^{25}$ and Kalichman et $\mathrm{al}^{27}$, who did find associations between BMI and functional outcomes.

In relation to the studies that reported a positive relation, Nishioka et al. ${ }^{21}$ used a 2 -level categorical variable (obesity - yes/no) $(\mathrm{BMI} \geq 27.5 \mathrm{~kg} / \mathrm{m} 2)$. Their multivariate linear regression adjusted by sex, LOS, TSO and TFIM at admission, when using TFIM at discharge as dependent variable, reported an $\mathrm{R}^{2}=0.66$. In our case using the same variables except TSO and sex (which were not significantly correlated to TFIM at discharge as 
presented in Table 3) and further including NIHSS, dysphagia, depression and ataxia (significantly correlated to TFIM at discharge) our reported $\mathrm{R}^{2}=0.4813$. Nishioka et al did not adjust the model for stroke severity (NIHSS not reported) ${ }^{21}$.

The other study that reported a positive relation, Morone et $\mathrm{al}^{22}$ concluded that increased BMI was correlated with improved rehabilitation effectiveness as reflected by the Barthel Index. Effectiveness of rehabilitation was significantly correlated with BMI at discharge (Spearman's $r=0.111)$. NIHSS was not reported ${ }^{22}$. In our case we did not assess participants using Barthel Index, nevertheless Barthel Index and FIM are highly correlated ${ }^{29}$.

In our study we have $68 \%$ men and only $32 \%$ women, meanwhile when comparing with both studies with a positive relation between BMI and functionality, the mean age of participants in Morone et al study was 68 years old ${ }^{22}$ and 72 years old ${ }^{21}$ in Nishioka et al. Besides, in both studies the proportion of women and men is quite similar (46\% and 54\%). (Details are presented in supplementary material Table SM3). After the age of 60, average body weight and muscle mass tend to decrease. As physical activity and energy expenditure also decrease there is a tendency to fat accumulation and fat redistribution ${ }^{30}$.

Kalichman et $\mathrm{al}^{27}$ reported a statistically significant negative correlation between TFIM gain and BMI in the total sample. In Appendix we present correlations analysis in the total sample, in our case BMI was not correlated to any FIM outcome, in particular TFIM gain, CFIM gain neither MFIM gain.

Stroke severity at admission in Kalichman et $\mathrm{al}^{27}$ was NIHSS $=8.03 \pm 4.38$, meanwhile in our case NIHSS severity was higher $(12.80 \pm 5.37)$. In our sample, when categorizing stroke severity using RPG benchmark, half of participants were categorized as RPG-severe (the percentage of RPG-severe patients across BMI categories was $51.7 \%$ as presented in Table 1). In the case of obese patients, almost $60 \%$ of them were RPG-severe in our sample. 
In relation to mean age, Kalichman ${ }^{27}$ reported $63.07 \pm 10.47$, in our case it was $49.63 \pm 9.32$.

Kalichman ${ }^{27}$ performed adjustments using linear regression for age, but not for severity.

Burke et $\mathrm{al}^{25}$ provide adjusted means for the MFIM efficiency and CFIM efficiency subscores by weight category. After adjusting for age and sex, the MFIM efficiency did not significantly differ by BMI subgroups $(\mathrm{p}=0.17)$. These results are similar to ours, as reported in Table 5 model 1 ( $\mathrm{p}=0.949)$. Nevertheless, after adjusting for age and sex, CFIM efficiency differed by BMI subgroups according to Burke results $(\mathrm{p}=0.01)^{25}$ but not in our case Table 5 model 3 ( $\mathrm{p}=0.771$ ). In our case we further adjusted by NIHSS severity and dysphagia but did not significantly differ either (Table 5 model 4).

We performed Bivariate Spearman's correlation analysis considering each BMI category separately and identified different associations within BMI categories. Aside from those presented in Table 6 for TFIM, further associations are detailed in Supplementary Material Table SM2 for MFIM. For example, there was no significant association for overweight patients between NIHSS and MFIM discharge, MFIM gain, neither MFIM effectiveness, but NIHSS was strongly associated to MFIM discharge in obese and normal weight patients.

Similarly, LOS was strongly associated to MFIM efficiency for obese patients, but there was no association for overweight patients and the association is weak for normal weight patients. MFIM at admission is not associated to MFIM efficiency for obese patients, but it is for normal and overweight patients. Similar behavior is observed for the CFIM subscale.

Finally it is important to remark that BMI assessment as the sole indicator of stroke functional recovery may present some limits as this parameter does not allow a proper evaluation of the distribution of fat mass and fat free mass, and thus makes the evaluation of body composition too simplistic ${ }^{31}$. 
As reported in recent previous research underweight patients with type 2 diabetes mellitus (T2DM) consistently showed poor outcomes when compared to obese patients ${ }^{32}$. Previous studies also showed poor outcomes in underweight T2DM patients. The patients who have a greater genetic susceptibility to T2DM have a greater chance of developing T2DM at lower BMI, which will consequently lead to a poor prognosis ${ }^{33}$ In relation to participants with T2DM in our study (20\% of our included patients) we found significant differences when comparing overweight and normal weight participants in relation to their total FIM Efficiency (0.342 (0.153) in normal weight and $0.656(0.315)$ in overweight patients, $p=0.032)$ but these results must be interpreted with caution because in our study normal weight patients with T2DM were only $n=9$ and overweight patients with T2DM were only $n=18$.

In relation to recurrent stroke, lower mortality and lower risk of readmission for recurrent stroke has been previously reported in obese patients ${ }^{34}$, the contribution of our study in this direction is limited because only $6.7 \%$ of participants had a second stroke.

Risk of death by stroke has been also associated to BMI in previous research ${ }^{35}$ but only $3.43 \%$ of our sample died in the period under study. A reason for this can be found in the young mean age of our sample.

\subsection{Study Limitations}

The data for this study was collected in one single tertiary center, suggesting that the generalization of these results should be considered carefully. Nevertheless, patients' severity assessed by means of NIHSS, the BMI categories determined following WHO classification, the RPG benchmark used to assess functional severity and the focus on working-age population, allows for similar comparative studies. 
A large number of stroke patients were not eligible for this study due to missing assessments, no data imputation was performed in this study, if any assessment was missing the patient was not included in the final analysis.

The specific manner in which stroke rehabilitation services are delivered (locally and internationally) may also impact outcomes. Therefore, the relationship between obesity and functional recovery after stroke could have been impacted by these changes along time, in the center where this study took place and similarly in related clinical rehabilitation centers, as also remarked in previous research (e.g. MacDonald et $\mathrm{al}^{5}$ ).

Adjusted $\mathrm{R}^{2}$ was confirmed by means of 10 -fold cross validation repeated ten times, in a test set, we independently partitioned initial data in training set (65\%) and test set (35\%), nevertheless results may require an external validation.

A further limitation is the measurement of body weight using BMI. It has been criticized for being unable to discriminate between fat and lean mass ${ }^{36}$ and also because its diagnostic performance worsened with increasing age ${ }^{36}$. Nevertheless our analysis is focused on working-age adults (about twenty year younger in mean age than related research presented in MacDonald's et al systematic review ${ }^{5}$ ).

\section{Conclusions}

Several variables were analyzed in this work to assess their potential impact regarding the association between BMI and the functional progress of working-age, mostly severe, firstever or recurrent patients with stroke, admitted to a rehabilitation hospital.

We found no associations between BMI and FIM measures (at discharge, admission, gain, efficiency or effectiveness). 
BMI was not found as significant predictor of FIM at discharge, either of FIM efficiency, even after adjusting using state-of-the art variables neither when using variables (diabetes, depression, dysphagia, or stroke severity) that have scarcely been used in previous studies addressing BMI and functionality.

We performed Spearman's correlation analyses separately for normal weight, overweight and obese patients and in that case we were able to identify associations that exist in one BMI category that do not exist in the others, for TFIM and also for CFIM and MFIM. 


\section{References}

1. Forlivesi S, Cappellari M, Bonetti B. Obesity paradox and stroke: a narrative review. Eat Weight Disord. 2020 Mar.

2. Meschia JF, Bushnell C, Boden-Albala B et al (2014) Guidelines for the primary prevention of stroke: a statement for healthcare professionals from the American Heart Association/American Stroke Association. Stroke 45:3754-3832.

3. Gruberg L, Weissman NJ, Waksman R et al (2002) The impact of obesity on the short-term and long-term outcomes after percutaneous coronary intervention: the obesity paradox? J Am Coll Cardiol 39:578-584.

4. Bouslama M, Perez H, Barreira C, Haussen D, Grossberg J, Belagaje S, Bianchi N, Anderson A, MFrankel M, Nogueira R, Body Mass Index and Clinical Outcomes in Large Vessel Occlusion Acute Ischemic Stroke after Endovascular Therapy. Intervent Neurol 2019;8:144-151.

5. MacDonald S, Journeay W and Uleryk E (2020). A systematic review of the impact of obesity on stroke inpatient rehabilitation functional outcomes. NeuroRehabilitation. 1-13. 10.3233/NRE-192979. 
6. Lau LH, Lew J, Borschmann K, Thijs V, Ekinci EI. Prevalence of diabetes and its effects on stroke outcomes: A meta-analysis and literature review. J Diabetes Investig. 2019;10(3):780-792.

7. Paolucci S, Iosa M, Coiro P, Venturiero V, Savo A, De Angelis D and Morone G (2019) Post-stroke Depression Increases Disability More Than $15 \%$ in Ischemic Stroke Survivors: A Case-Control Study. Front. Neurol. 10:926.

8. Karataş, M. et al. Functional outcome in stroke patients with atrial fibrillation Archives of Physical Medicine and Rehabilitation (2000), Volume 81, Issue 8, 1025 1029.

9. Choi SW, Han N, Jung SH, Kim HD, Eom MJ, Bae HW. Evaluation of Ataxia in Mild Ischemic Stroke Patients Using the Scale for the Assessment and Rating of Ataxia (SARA). Ann Rehabil Med. 2018;42(3):375-383. Published 2018 Jun 27.

10. Huang KL, Liu T, Yu-Chi H and Pong Y. (2014). Functional Outcome in Acute Stroke Patients with Oropharyngeal Dysphagia after Swallowing Therapy. Journal of Stroke and Cerebrovascular Diseases. 23. 10.1016/j.jstrokecerebrovasdis.2014.05.031

11. Kang J, Ko Y, Park JH, et al. Effect of blood pressure on 3-month functional outcome in the subacute stage of ischemic stroke. Neurology. 2012;79(20):2018-2024. 
12. Ng, Y., Tan, K \& Chen, Cynthia \& Koh, Gerald. (2016). How Do Recurrent and First-Ever Strokes Differ in Rehabilitation Outcomes?. American journal of physical medicine \& rehabilitation / Association of Academic Physiatrists. 95.

13. Ekker, MS, Verhoeven, JI, Vaartjes, I, et al. Stroke incidence in young adults according to age, subtype, sex, and time trends. Neurology 2019; 92: e2444-e2454.

14. Putaala J. Ischemic stroke in the young: Current perspectives on incidence, risk factors, and cardiovascular prognosis. Eur Stroke J 2016 Mar 08;1(1):28-40

15. Towfighi A, Ovbiagele B: The impact of body mass index on mortality after stroke. Stroke 2009;40:2704-8

16. https://strobe-statement.org/index.php?id=available-checklists

17. Shah S, Vanclay F, Cooper B. Efficiency, effectiveness, and duration of stroke rehabilitation. Stroke. 1990 Feb;21(2):241-6.

18. Lai W, Buttineau M, Harvey J, Pucci R, Wong A, Dell'Erario L, Bosnyak S., Reid, S and Salbach N. (2017). Clinical and psychosocial predictors of exceeding target length of stay during inpatient stroke rehabilitation. Topics in Stroke Rehabilitation. 24. 1-7.

19. Uswatte G, Taub E. Constraint-induced movement therapy: a method for harnessing neuroplasticity to treat motor disorders. Prog Brain Res. 2013;207:379-401.

20. O’Brien RM (2007). "A Caution Regarding Rules of Thumb for Variance Inflation Factors". Quality \& Quantity. 41 (5): 673-690. 
21. Nishioka S., Wakabayashi H., Yoshida T., Mori N., Watanabe R and Nishioka E. (2016). Obese Japanese patients with stroke have higher functional recovery in convalescent rehabilitation wards: A retrospective cohort study. J Stroke Cerebrovasc Dis 25, 26-33.

22. Morone, G, Iosa M., Paolucci T., Muzzioli L and Paolucci S. (2019). Relationship between body mass index and rehabilitation outcomes in subscute stroke with dysphagia. Am J Phys Med Rehabil, 98, 608-612.

23. Hagii J, Metoki N, Saito S, Fujita A, Shiroto H and Sasaki S (2018). Low body mass index is a poor prognosis factor in cardioembolic stroke patients with nonvalvular atrial fibrillation. J Stroke Cerebrovasc Dis, 27, 3155-3162.

24. Karaahmet OZ, Umay E, Gurcay E., Serce A., Gundogdu I and Cakci A. (2018). The effect of premorbid features on post-stroke rehabilitation outcome. Iran J Neurol, 17, $38-46$.

25. Burke DT., Al-Adawi S, Bell RB, Easley K, Chen S and Burke DP. (2014). Effect of body mass index on stroke 485 rehabilitation. Arch Phys Med Rehabil, 95, 10551059.

26. Kalichman L, Rodrigues B, Gurvich D, Israelov, Z and Spivak E. (2007). Impact of patient's weight on stroke rehabilitation results. Am J Phys Med Rehabil, 86, 650655.

27. Kalichman L, Alperovitch-Najenson D and Treger I. (2016). The impact of patient's weight on post-stroke rehabilitation. Disabil Rehabil, 38, 1684-1690. 
28. Rankin J. (1957). Cerebral vascular accidents in patients over the age of 60. II. Prognosis. Scott Med J, 2, 200-215.

29. Kwon S. Hartzema P, Min-Lai A. Disability Measures in Stroke Relationship Among the Barthel Index, the Functional Independence Measure, and the Modified Rankin Scale. 2004;35:918-923

30. Kennedy RL, Chokkalingham K, Srinivasan R. Obesity in the elderly: who should we be treating, and why, and how? Curr Opin Clin Nutr Metab Care. 2004 Jan;7(1):3-9.

31. Ciancarelli I, Tonin P, Garo ML, Tozzi Ciancarelli MG. Effectiveness of intensive neurorehabilitation in obese subacute stroke patients. Funct Neurol. 2019 JanMar;34(1):45-51.

32. Park H, Lee HW, Yoo J, Lee HS, Nam HS, Kim YD, Heo JH. Body Mass Index and Prognosis in Ischemic Stroke Patients With Type 2 Diabetes Mellitus. Front Neurol. 2019 Jun 5;10:563.

33. Costanzo P, Cleland JG, Pellicori P, Clark AL, Hepburn D, Klipatrick ES, et al. The obesity paradox in type 2 diabetes mellitus: Relationship of body mass index to prognosis: a cohort study. Ann Intern Med. (2015) 162:610-8.

34. Andersen KK, Olsen TS. The obesity paradox in stroke: lower mortality and lower risk of readmission for recurrent stroke in obese stroke patients. Int J Stroke. 2015 Jan;10(1):99-104. 
35. Dehlendorff C, Andersen KK, Olsen TS. Body mass index and death by stroke: no obesity paradox. JAMA Neurol. 2014 Aug;71(8):978-84.

36. Romero-Corral A, Somers VK, Sierra-Johnson J et al (2008) Accuracy of body mass index in diagnosing obesity in the adult general population. Int J Obes. 32:959-966. 
Table 1. Characteristics at admission

\begin{tabular}{|c|c|c|c|c|c|}
\hline & $\begin{array}{l}\text { Normal } \\
(N=84)\end{array}$ & $\begin{array}{l}\text { Overweight } \\
(\mathbf{N}=59)\end{array}$ & $\begin{array}{l}\text { Obese } \\
(N=35)\end{array}$ & $\begin{array}{l}\text { TOTAL } \\
(\mathrm{N}=178)\end{array}$ & $\mathbf{p}$ \\
\hline Sex (females) & $29(34.5 \%)$ & $10(16.9 \%)$ & $17(48.6 \%)$ & $56(31.5 \%)$ & 0.004 \\
\hline Age in years, mean (SD) & $48.47(10.65)$ & $50.71(7.74)$ & $50.59(8.18)$ & $49.63(9.32)$ & 0.663 \\
\hline \multicolumn{5}{|l|}{ Type of stroke } & \multirow[t]{3}{*}{0.593} \\
\hline ischemic & $63(75.0 \%)$ & $40(67.8 \%)$ & $24(68.6 \%)$ & $127(71.3 \%)$ & \\
\hline hemorrhagic & $21(25.0 \%)$ & $19(32.2 \%)$ & $11(31.4 \%)$ & $51(28.7 \%)$ & \\
\hline \multicolumn{5}{|l|}{ OCSP Classification } & \multirow{5}{*}{0.294} \\
\hline LACI & $1(1.2 \%)$ & $1(1.7 \%)$ & $1(2.9 \%)$ & $3(1.7 \%)$ & \\
\hline POCI & $9(10.7 \%)$ & $1(1.7 \%)$ & $0(0.0 \%)$ & $10(5.6 \%)$ & \\
\hline TACI & $46(54.8 \%)$ & $33(55.9 \%)$ & $19(54.3 \%)$ & $98(55.1 \%)$ & \\
\hline PACI & $7(8.3 \%)$ & $5(8.5 \%)$ & $4(11.4 \%)$ & $16(9.0 \%)$ & \\
\hline \multicolumn{5}{|l|}{ Hemorrhagic subtypes } & \multirow[t]{3}{*}{0.294} \\
\hline Primary & $14(16.7 \%)$ & $10(16.9 \%)$ & $9(25.7 \%)$ & $33(18.5 \%)$ & \\
\hline Secondary & $7(8.3 \%)$ & $9(15.3 \%)$ & $2(5.7 \%)$ & $18(10.1 \%)$ & \\
\hline NIHSS, mean (SD) & $12.96(5.49)$ & $12.40(5.44)$ & $13.08(5.05)$ & $12.80(5.37)$ & 0.846 \\
\hline \multicolumn{5}{|l|}{ RPG stroke Severity } & \multirow{4}{*}{0.237} \\
\hline Mild-RPG & $12(14.3 \%)$ & $6(10.2 \%)$ & $8(22.9 \%)$ & $26(14.6 \%)$ & \\
\hline Moderate-RPG & $29(34.5 \%)$ & $24(40.7 \%)$ & $7(20.0 \%)$ & $60(33.7 \%)$ & \\
\hline Severe-RPG & $43(51.2 \%)$ & $29(49.2 \%)$ & $20(57.1 \%)$ & $92(51.7 \%)$ & \\
\hline Time since onset to Adm & $17.50(4.84)$ & $18.06(4.22)$ & $17.94(4.50)$ & $17.76(4.59)$ & 0.925 \\
\hline BMI Adm & $22.33(1.76)$ & $27.06(1.34)$ & $32.15(1.65)$ & $25.83(4.09)$ & $<0.001$ \\
\hline \multicolumn{5}{|l|}{ Smoking habits } & \multirow{4}{*}{0.078} \\
\hline Current & $20(23.8 \%)$ & $10(16.9 \%)$ & $5(14.3 \%)$ & $35(19.7 \%)$ & \\
\hline Former & $3(3.6 \%)$ & $9(15.3 \%)$ & $6(17.1 \%)$ & $18(10.1 \%)$ & \\
\hline Non & $61(72.6 \%)$ & $40(67.8 \%)$ & $24(68.6 \%)$ & $125(70.2 \%)$ & \\
\hline Hypertension & $50(59.5 \%)$ & $41(69.5 \%)$ & $27(77.1 \%)$ & $118(66.3 \%)$ & 0.147 \\
\hline Hyperlipidemia & $26(31.0 \%)$ & $21(35.6 \%)$ & $13(37.1 \%)$ & $60(33.7 \%)$ & 0.754 \\
\hline Dysphagia & $29(34.5 \%)$ & $15(25.4 \%)$ & $10(28.6 \%)$ & $54(30.3 \%)$ & 0.491 \\
\hline Medication for depression & $43(51.2 \%)$ & $33(55.9 \%)$ & $22(62.9 \%)$ & $98(55.1 \%)$ & 0.500 \\
\hline Diabetes & $9(10.7 \%)$ & $18(30.5 \%)$ & $9(25.7 \%)$ & $36(20.2 \%)$ & 0.01 \\
\hline Atrial Fibrillation & $6(7.1 \%)$ & $9(15.3 \%)$ & $2(5.7 \%)$ & $17(9.6 \%)$ & 0.184 \\
\hline Ataxia & $7(8.3 \%)$ & $2(3.4 \%)$ & $0(0.0 \%)$ & $9(5.1 \%)$ & 0.130 \\
\hline Recurrent stroke & $6(7.1 \%)$ & $3(5.1 \%)$ & $3(8.6 \%)$ & $12(6.7 \%)$ & 0.792 \\
\hline Falls & $24(28.6 \%)$ & $16(27.1 \%)$ & $17(48.6 \%)$ & $57(32.0 \%)$ & 0.063 \\
\hline \multicolumn{5}{|l|}{ Educational level } & \multirow{4}{*}{0.118} \\
\hline Primary & $46(54.8 \%)$ & $24(40.7 \%)$ & $15(42.9 \%)$ & $85(47.8 \%)$ & \\
\hline Intermmediate & $18(21.4 \%)$ & $21(35.6 \%)$ & $15(42.9 \%)$ & $54(30.3 \%)$ & \\
\hline Higher & $20(23.8 \%)$ & $14(23.7 \%)$ & $5(14.3 \%)$ & $39(21.9 \%)$ & \\
\hline LOS in days & $61.19(27.38)$ & $59.56(24.81)$ & $61.31(28.99)$ & $60.67(26.74)$ & 0.906 \\
\hline
\end{tabular}

All characteristics are presented as frequencies and percentages, $\mathrm{n}(\%)$, unless otherwise indicated. SD: standard deviation;

Oxfordshire Community Stroke Project (OCSP).

total anterior circulation infarcts (TACI), partial anterior circulation infarcts (PACI), lacunar infarcts (LACI), and posterior circulation infarcts (POCI); 
NIHSS: National Institutes of Health Stroke Scale; FIM: Functional Independence; LOS = Length of Stay; BMI=Body Mass Index; RPG=Rehabilitation Patient Groups 
Table 2. Functional assessments at admission and discharge between individuals in different BMI groups

\begin{tabular}{|c|c|c|c|c|c|}
\hline & \begin{tabular}{|l} 
Normal \\
$(\mathbf{N}=84)$
\end{tabular} & $\begin{array}{l}\text { Overweight } \\
(\mathrm{N}=59)\end{array}$ & $\begin{array}{l}\text { Obese } \\
(\mathrm{N}=35)\end{array}$ & \begin{tabular}{|l} 
TOTAL \\
$(\mathbf{N}=178)$
\end{tabular} & $\mathbf{p}$ \\
\hline T-FIM Adm & $60.69(28.02)$ & $64.712(21.733)$ & $61.45(26.36)$ & $62.174(25.688)$ & 0.592 \\
\hline C- FIM Adm & $20.893(10.516)$ & $23.966(7.697)$ & $24.714(9.596)$ & $22.663(9.583)$ & 0.105 \\
\hline M-FIM Adm & $39.798(21.470)$ & $40.746(18.083)$ & $36.743(19.440)$ & 39.511 (19.949) & 0.557 \\
\hline T-FIM Dis & $95.786(22.343)$ & $96.712(16.833)$ & 93.743 (23.919) & 95.691 (20.927) & 0.857 \\
\hline C- FIM Dis & $25.988(8.789)$ & $28.186(6.482)$ & $28.943(7.211)$ & $27.298(7.848)$ & 0.229 \\
\hline M-FIM Dis & $69.798(16.066)$ & $68.525(14.311)$ & $64.800(19.888)$ & $68.393(16.359)$ & 0.436 \\
\hline T-FIM Gain & $35.095(21.952)$ & $32.000(16.806)$ & $32.286(19.769)$ & 33.517 (19.896) & 0.722 \\
\hline C- FIM Gain & $5.095(6.156)$ & $4.220(4.602)$ & $4.229(4.544)$ & $4.635(5.373)$ & 0.967 \\
\hline M-FIM Gain & $30.000(17.842)$ & $27.780(15.546)$ & 28.057 (18.096) & $28.882(17.103)$ & 0.667 \\
\hline T-FIM Efficiency median $\left(\mathrm{P}_{25}-\mathrm{P}_{75}\right)$ & $0.518(0.311,0.881)$ & $0.568(0.351,0.709)$ & $0.569(0.408,0.834)$ & $0.551(0.345,0.813)$ & 0.936 \\
\hline T-FIM Efficiency mean & $0.639(0.455)$ & $0.575(0.299)$ & $0.611(0.378)$ & $0.612(0.393)$ & 0.936 \\
\hline C-FIM Efficiency median $\left(\mathrm{P}_{25}-\mathrm{P}_{75}\right)$ & $0.043(0.000,0.129)$ & $0.062(0.018,0.109)$ & $0.060(0.000,0.111)$ & $0.054(0.000,0.125)$ & 0.948 \\
\hline C-FIM Efficiency mean & $0.084(0.101)$ & $0.073(0.080)$ & $0.075(0.084)$ & $0.078(0.091)$ & 0.948 \\
\hline M-FIM Efficiency median $\left(\mathrm{P}_{25}-\mathrm{P}_{75}\right)$ & $0.452(0.276,0.802)$ & $0.515(0.305,0.660)$ & $0.506(0.358,0.726)$ & $0.481(0.292,0.729)$ & 0.907 \\
\hline M-FIM Efficiency mean & $0.555(0.413)$ & $0.503(0.284)$ & $0.536(0.344)$ & $0.534(0.360)$ & 0.907 \\
\hline C-FIM Effectiveness median $\left(\mathrm{P}_{25}-\mathrm{P}_{75}\right)$ & $26.50(0.00,61.16)$ & $31.818(8.33,66.73)$ & $34.78(0.00,65.15)$ & $31.534(0.00,63.35)$ & 0.591 \\
\hline C-FIM Effectiveness mean & $34.028(35.497)$ & $37.647(39.509)$ & $39.206(32.782)$ & $36.246(36.243)$ & 0.591 \\
\hline M-FIM Effectiveness median $\left(\mathrm{P}_{25}-\mathrm{P}_{75}\right)$ & $62.12(44.07,76.99)$ & $56.25(39.05,75.00)$ & $54.16(35.18,72.12)$ & $58.27(38.97,75.38)$ & 0.314 \\
\hline M-FIM Effectiveness mean & $58.255(26.323)$ & $55.917(25.128)$ & $50.868(27.562)$ & $56.027(26.179)$ & 0.314 \\
\hline
\end{tabular}

All assessments are presented as mean (SD), unless otherwise indicated

FIM gain definition: difference between FIM admission and FIM discharge.

FIM efficiency definition: FIM gain divided by LOS;

FIM: Functional Independence Measure; T-FIM: Total FIM; M-FIM: Motor FIM; C-FIM: Cognitive FIM;

T-FIM=M-FIM+C-FIM; LOS = Length of Stay This document is protected by international copyright laws. No additional reproduction is authorized. It is permitted for personal use to download and save only one file and print only one
copy of this Article. It is not permitted to make additional copies (either sporadically or systematically, either printed or electronic) of the Article for any purpose. It is not permitted to distribute
the electronic copy of the article through online internet and/or intranet file sharing systems, electronic mailing or any other means which may allow access to the Article. The use of all or any

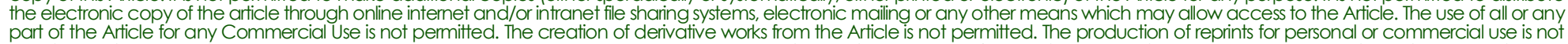
permitted. It is not permitted to remove, cover, overlay, obscure, block, or change any copyright nofices or terms of use which the Publisher may post on the Article. It is not permitted to 
Table 3. Correlation analysis (BMI as continuous variable)

\begin{tabular}{|l|l|l|l|l|l|l|}
\hline & \multicolumn{2}{l}{ T-FIM Dis } & \multicolumn{2}{l|}{ C-FIM effi } & \multicolumn{2}{l|}{ M-FIM Effi } \\
\hline & rho & $\mathbf{p}$ & rho & $\mathbf{p}$ & rho & $\mathbf{p}$ \\
\hline Age in years & -0.165 & $\mathbf{0 . 0 2 7}$ & 0.102 & 0.174 & -0.154 & $\mathbf{0 . 0 3 9}$ \\
\hline Sex & 0.020 & 0.786 & -0.094 & 0.211 & 0.063 & 0.398 \\
\hline NIHSS Acute & -0.543 & $<\mathbf{0 . 0 0 1}$ & 0.169 & $\mathbf{0 . 0 2 3}$ & -0.021 & 0.780 \\
\hline TSO & -0.074 & 0.321 & 0.040 & 0.595 & -0.009 & 0.896 \\
\hline Hypertension & -0.124 & 0.097 & -0.003 & 0.966 & -0.009 & 0.899 \\
\hline Dysphagia & -0.183 & $\mathbf{0 . 0 1 4}$ & 0.189 & $\mathbf{0 . 0 1 1}$ & -0.033 & 0.659 \\
\hline Depression & -0.163 & $\mathbf{0 . 0 2 8}$ & 0.135 & 0.071 & 0.031 & 0.674 \\
\hline Diabetes & -0.072 & 0.337 & 0.044 & 0.556 & -0.066 & 0.377 \\
\hline Hyperlipidemia & 0.008 & 0.912 & 0.068 & 0.364 & 0.010 & 0.890 \\
\hline Ataxia & 0.158 & $\mathbf{0 . 0 3 4}$ & 0.003 & 0.967 & 0.034 & 0.651 \\
\hline Atrial fibrillation & -0.015 & 0.839 & 0.072 & 0.337 & 0.091 & 0.226 \\
\hline Recurrent stroke & -0.006 & 0.928 & -0.087 & 0.246 & -0.011 & 0.879 \\
\hline Smoking habits & -0.042 & 0.570 & 0.065 & 0.383 & -0.028 & 0.708 \\
\hline BMI Adm & -0.085 & 0.256 & -0.008 & 0.915 & -0.007 & 0.916 \\
\hline Years of education & 0.070 & 0.35 & 0.029 & 0.692 & -0.110 & 0.140 \\
\hline T-FIM Adm & 0.636 & $<\mathbf{0 . 0 0 1}$ & -0.334 & $<\mathbf{0 . 0 0 1}$ & -0.211 & $\mathbf{0 . 0 0 4}$ \\
\hline C-FIM Adm & 0.569 & $<\mathbf{0 . 0 0 1}$ & -0.538 & $<\mathbf{0 . 0 0 1}$ & 0.068 & 0.366 \\
\hline M-FIM Adm & 0.560 & $<\mathbf{0 . 0 0 1}$ & -0.205 & $\mathbf{0 . 0 0 6}$ & -0.285 & $\mathbf{0 . 0 0 0 1}$ \\
\hline Length of Stay & -0.324 & $<\mathbf{0 . 0 0 1}$ & 0.0367 & 0.626 & -0.308 & $<\mathbf{0 . 0 0 1}$ \\
\hline
\end{tabular}

NIHSS: National Institutes of Health Stroke Scale; FIM: Functional Independence Measure;

T-FIM: Total FIM; M-FIM: Motor FIM; C-FIM: Cognitive FIM;

T-FIM=M-FIM+C-FIM; 
Table 4. Multivariate lineal regressions, non standard beta 95\% CIs, standard beta, Durbin test, VIF, $\mathrm{R}^{2}$ and Adjusted $\mathrm{R}^{2}$

\begin{tabular}{|c|c|c|c|c|c|c|c|c|c|}
\hline Model & Variables & $\beta(95 \% \mathrm{CI})$ & Std $\beta$ & VIF & Tol & $\mathbf{p}$ & $\mathbf{R}^{2}$ & Adj $R^{2}$ & 10FCVR10 \\
\hline \multirow{8}{*}{$\begin{array}{l}1 \\
\text { TFIMDis }\end{array}$} & NIHSS & $-0.810(-1.348,-0.272)$ & -0.208 & 1.60 & 0.62 & 0.003 & \multirow[t]{8}{*}{0.4813} & \multirow[t]{8}{*}{0.46} & \multirow[t]{8}{*}{0.4802} \\
\hline & Age & $-0.221(-0.471,0.027)$ & -0.098 & 1.03 & 0.96 & 0.080 & & & \\
\hline & Dysphagia & $0.406(-4.847,5.660)$ & 0.008 & 1.12 & 0.88 & 0.878 & & & \\
\hline & Depression & $3.053(-1.891,7.998)$ & 0.072 & 1.16 & 0.85 & 0.224 & & & \\
\hline & Ataxia & $10.252(-0.403,20.907)$ & 0.107 & 1.05 & 0.94 & 0.059 & & & \\
\hline & LOS & $-0.017(-0.117,0.083)$ & -0.022 & 1.38 & 0.72 & 0.733 & & & \\
\hline & TFIMAdm & $0.430(0.305,0.555)$ & 0.528 & $\mathbf{1 . 9 7}$ & 0.50 & $<0.001$ & & & \\
\hline & Durbin test & \multicolumn{5}{|l|}{$\mathrm{D}-\mathrm{W}=1.965 ; \mathrm{p}=0.768$} & & & \\
\hline \multirow{5}{*}{$\begin{array}{l}2 \\
\text { TFIMDis }\end{array}$} & Age & $-0.223(-0.483,0.036)$ & -0.099 & 1.06 & 0.93 & 0.091 & \multirow[t]{5}{*}{0.4427} & \multirow[t]{5}{*}{0.4298} & \multirow[t]{5}{*}{0.4412} \\
\hline & Sex & $-0.203(-5.299,4.893)$ & -0.004 & 1.02 & 0.97 & 0.937 & & & \\
\hline & BMI & $-0.331(-0.914,0.252)$ & -0.064 & 1.04 & 0.96 & 0.264 & & & \\
\hline & TFIMAdm & $0.525(0.433,0.616)$ & 0.644 & 1.00 & 0.99 & $<0.001$ & & & \\
\hline & Durbin test & \multicolumn{5}{|l|}{$\mathrm{D}-\mathrm{W}=1.943 ; \mathrm{p}=0.636$} & & & \\
\hline
\end{tabular}

NIHSS: National Institutes of Health Stroke Scale

FIM: Functional Independence Measure; C-FIM: Cognitive FIM; T-FIM=M-FIM+C-FIM;

BMI: Body Mass Index, LOS: Length of Stay; 10FCVR10: 10-fold cross validation repeated 10 times 
Table 5. Motor and Cognitive FIM efficiency by BMI categories.

\begin{tabular}{|c|c|c|c|c|c|c|}
\hline & $\begin{array}{l}\text { BMI } \\
\text { Categories }\end{array}$ & $\mathbf{n}$ & $\begin{array}{l}\text { Mean MFIM } \\
\text { Efficiency }\end{array}$ & $95 \%$ CI lr & $95 \%$ CI upr & se \\
\hline \multirow{4}{*}{$\begin{array}{l}\text { Model } 1 \\
\text { Adj: } \\
\text { age and sex }\end{array}$} & Normal & 84 & 0.541 & 0.465 & 0.617 & 0.038 \\
\hline & Overweight & 59 & 0.521 & 0.429 & 0.613 & 0.046 \\
\hline & Obese & 35 & 0.536 & 0.417 & 0.655 & 0.060 \\
\hline & \multicolumn{6}{|l|}{$\mathrm{P}=0.949$} \\
\hline \multirow{4}{*}{$\begin{array}{l}\text { Model } 2 \\
\text { Adj: } \\
\text { age and } \\
\text { LOS }\end{array}$} & Normal & 84 & 0.540 & 0.476 & 0.605 & 0.032 \\
\hline & Overweight & 59 & 0.518 & 0.441 & 0.595 & 0.038 \\
\hline & Obese & 35 & 0.542 & 0.443 & 0.642 & 0.0502 \\
\hline & \multicolumn{6}{|l|}{$\mathrm{P}=0.890$} \\
\hline & $\begin{array}{l}\text { BMI } \\
\text { Categories }\end{array}$ & $\mathbf{n}$ & $\begin{array}{l}\text { Mean CFIM } \\
\text { Efficiency }\end{array}$ & $95 \%$ CI lr & $95 \%$ CI upr & se \\
\hline \multirow{4}{*}{$\begin{array}{l}\text { Model } 3 \\
\text { Adj: } \\
\text { Age and sex }\end{array}$} & Normal & 84 & 0.083 & 0.064 & 0.102 & 0.009 \\
\hline & Overweight & 59 & 0.072 & 0.049 & 0.095 & 0.011 \\
\hline & Obese & 35 & 0.077 & 0.047 & 0.107 & 0.015 \\
\hline & \multicolumn{6}{|l|}{$\mathrm{P}=0.771$} \\
\hline \multirow{4}{*}{$\begin{array}{l}\text { Model } 4 \\
\text { Adj: } \\
\text { NIHSS and } \\
\text { dysphagia }\end{array}$} & Normal & 84 & 0.081 & 0.062 & 0.100 & 0.009 \\
\hline & Overweight & 59 & 0.075 & 0.053 & 0.098 & 0.014 \\
\hline & Obese & 35 & 0.075 & 0.046 & 0.104 & 0.011 \\
\hline & \multicolumn{6}{|l|}{$\mathrm{P}=0.902$} \\
\hline
\end{tabular}

NIHSS: National Institutes of Health Stroke Scale, MFIM: Motor FIM, CFIM: Cognitive

FIM, FIM: Functional Independence Measure; BMI: Body Mass Index, LOS: Length of Stay; 
Table 6. Spearman's correlations for normal weight group (top) overweight group (middle) and obesity group (bottom)

\begin{tabular}{|c|c|c|c|c|c|c|c|c|c|c|}
\hline & NIHSS & Age & TSO & BMI & LOS & TFIMAdm & TFIMDis & TFIMgain & TFIMeffi & TFIMEffe \\
\hline NIHSS & 1 & & & & & & & & & \\
\hline Age & ns & 1 & & & & & & & & \\
\hline TSO & ns & $\mathrm{ns}$ & 1 & & & & & & & \\
\hline BMI & ns & $0.41^{\mathrm{b}}$ & ns & 1 & & & & & & \\
\hline LOS & $0.31^{\mathrm{b}}$ & $0.22^{\mathrm{a}}$ & ns & ns & 1 & & & & & \\
\hline TFIMAdm & $-0.62^{b}$ & $\mathrm{~ns}$ & ns & $\mathrm{ns}$ & $-0.47^{b}$ & 1 & & & & \\
\hline TFIMDis & $-0.56^{\mathrm{b}}$ & $\mathrm{ns}$ & $\mathrm{ns}$ & $\mathrm{ns}$ & $-0.22^{\mathrm{a}}$ & $0.63^{\mathrm{b}}$ & 1 & & & \\
\hline TFIMgain & $0.26^{\mathrm{a}}$ & $\mathrm{ns}$ & ns & $\mathrm{ns}$ & $\mathbf{0 . 3 3}^{\mathrm{b}}$ & $-0.63^{b}$ & ns & 1 & & \\
\hline TFIMEffi & ns & $-0.26 \mathrm{a}$ & $\mathrm{ns}$ & $\mathrm{ns}$ & $-0.25^{a}$ & $-0.33^{b}$ & $0.23^{\mathrm{a}}$ & $0.79^{\mathrm{b}}$ & 1 & \\
\hline TFIMEffe & $0.26^{\mathrm{a}}$ & $\mathrm{ns}$ & $\mathrm{ns}$ & $\mathrm{ns}$ & $\mathbf{0 . 3 3}^{\mathrm{b}}$ & $-0.63^{b}$ & $\mathrm{~ns}$ & $1^{\mathrm{b}}$ & $0.79^{\mathrm{b}}$ & 1 \\
\hline & NIHSS & Age & TSO & BMI & LOS & TFIMAdm & TFIMDis & TFIMgain & TFIMeffi & TFIMEffe \\
\hline NIHSS & 1 & & & & & & & & & \\
\hline Age & ns & 1 & & & & & & & & \\
\hline TSO & ns & $\mathrm{ns}$ & 1 & & & & & & & \\
\hline BMI & ns & ns & ns & 1 & & & & & & \\
\hline LOS & $0.39^{\mathrm{b}}$ & $\mathrm{ns}$ & $\mathrm{ns}$ & $\mathrm{ns}$ & 1 & & & & & \\
\hline TFIMAdm & $-0.51^{b}$ & $\mathrm{~ns}$ & ns & $\mathrm{ns}$ & $-0.72^{b}$ & 1 & & & & \\
\hline TFIMDis & $-0.39^{b}$ & $\mathrm{~ns}$ & $-0.27^{\mathrm{a}}$ & $\mathrm{ns}$ & $-0.43^{b}$ & $0.61^{\mathrm{b}}$ & 1 & & & \\
\hline TFIMgain & ns & $\mathrm{ns}$ & ns & $\mathrm{ns}$ & $\mathbf{0 . 4 3}^{\mathrm{b}}$ & $-0.62^{b}$ & $\mathrm{~ns}$ & 1 & & \\
\hline TFIMEffi & ns & $\mathrm{ns}$ & $-0.30^{\mathrm{a}}$ & ns & ns & $\mathrm{ns}$ & $0.39^{\mathrm{b}}$ & $0.72^{\mathrm{b}}$ & 1 & \\
\hline TFIMEffe & $\mathrm{ns}$ & $\mathrm{ns}$ & ns & $\mathrm{ns}$ & $0.43^{b}$ & $-0.62^{b}$ & $\mathrm{~ns}$ & $1^{\mathrm{b}}$ & $0.72^{\mathrm{b}}$ & 1 \\
\hline & NIHSS & Age & TSO & BMI & LOS & TFIMAdm & TFIMDis & TFIMgain & TFIMeffi & TFIMEffe \\
\hline NIHSS & 1 & & & & & & & & & \\
\hline Age & ns & 1 & & & & & & & & \\
\hline TSO & ns & ns & 1 & & & & & & & \\
\hline BMI & $\mathrm{ns}$ & ns & ns & 1 & & & & & & \\
\hline LOS & $0.34^{\mathrm{b}}$ & ns & ns & ns & 1 & & & & & \\
\hline TFIMAdm & $-0.69^{b}$ & $-0.35^{\mathrm{a}}$ & ns & ns & $-0.53^{\mathrm{b}}$ & 1 & & & & \\
\hline TFIMDis & $-0.69^{\mathrm{b}}$ & $\mathrm{ns}$ & ns & $\mathrm{ns}$ & $-0.48^{\mathrm{b}}$ & $0.70^{\mathrm{b}}$ & 1 & & & \\
\hline TFIMgain & $\mathrm{ns}$ & $\mathrm{ns}$ & $\mathrm{ns}$ & $\mathrm{ns}$ & ns & $-0.43^{b}$ & $\mathrm{~ns}$ & 1 & & \\
\hline TFIMEffi & ns & ns & ns & ns & $-0.50^{b}$ & $\mathrm{~ns}$ & $0.34^{\mathrm{a}}$ & 0.73 & 1 & \\
\hline TFIMEffe & ns & ns & ns & ns & ns & $-0.43^{b}$ & ns & $1^{\mathrm{b}}$ & 0.73 & 1 \\
\hline
\end{tabular}

${ }^{a} \mathrm{P}<0.05$; ${ }^{\mathrm{b}} \mathrm{P}<0.01 ;$ ns: non-significant

NIHSS: National Institutes of Health Stroke Scale; TSO: time since stroke onset to rehabilitation admission;

BMI: Body Mass Index; LOS: length of stay;

FIM: Functional Independence Measure; TFIMAdm: total FIM at admission, TFIMDis:total FIM at discharge,

TFIMgain: total FIM gain, TFIMEffi: total FIM efficiency, TFIMEffe: total FIM efectiveness 


\section{Abbreviations}

BMI: Body Mass Index,

FIM: Functional Independence Measure,

C-FIM: Cognitive FIM subtest,

EHRs: Electronical Health Records,

ICD-9: International Classification of Diseases,

LACI: lacunar infarcts,

LOS: Length of stay,

M-FIM: Motor FIM subtest,

mRS: Modified Rankin Scale,

NIHSS: National Institute of Health Stroke Scale,

OCSP: Oxfordshire Community Stroke Project,

PACI: partial anterior circulation infarcts,

PMR test: Verbal fluency test, words starting with P, M, or R,

POCI: posterior circulation infarcts,

RPG: Rehabilitation Patient Groups,

STROBE: Strengthening the Reporting of Observational Studies in Epidemiology,

T-FIM: Total FIM test,

T2DM: Type 2 diabetes mellitus,

VIF: Variance Inflation Factor,

TACI: total anterior circulation infarcts,

WHO: World Health Organization. 


\section{Figure's Legends}

\section{Table's Legends}

Table 1. Characteristics at admission

Table 2. Functional assessments at admission and discharge between individuals in different

BMI categories

Table 3. Correlation analysis (BMI as continuous variable)

Table 4. Multivariate lineal regressions, non standard beta 95\% CIs, standard beta, Durbin test, VIF, R2 and Adjusted R2

Table 5. Motor and Cognitive FIM efficiency by BMI categories.

Table 6. Spearman's correlations for normal weight group (top) overweight group (middle) and obesity group (bottom) 


\section{Author's contributions:}

All authors read and approved the final version of the manuscript.

Study conception and design: Alejandro García-Rudolph, John D. Kelleher, Josep Maria

Tormos

Acquisition of data: Alejandro García-Rudolph, Blanca Cegarra, Joan Sauri

Analysis and interpretation of data: John D. Kelleher, Vasudevan Nedumpozhimana,

Alejandro García-Rudolph, Eloy Opisso

Drafting of manuscript: Alejandro García-Rudolph, Blanca Cegarra, Joan Sauri

Critical revision: John D. Kelleher, Montserrat Bernabeu, Josep Maria Tormos, Eloy Opisso 


\section{Supplementary Digital Material}

Download supplementary material file: Eur J Phys Rehabil Med-6411 Supplementary Digital Material5_V1_2020-10-01.pdf 


\section{Supplementary Digital Material}

Download supplementary material file: Eur J Phys Rehabil Med-6411 Supplementary Digital Material6 V1_2020-11-20.doc 